\title{
Meso-scale characterization of lithium distribution in Lithium-Ion batteries using ion beam analysis techniques.
}

R. Gonzalez-Arrabal ${ }^{1}$, M. Panizo-Laiz ${ }^{1}$, K. Fujita ${ }^{2}$, K. Mima ${ }^{2,3}$, A. Yamazaki ${ }^{4}$, T. Kamiya ${ }^{5}$, Y.

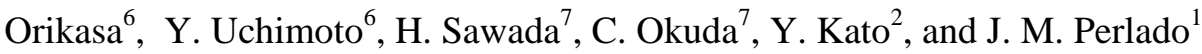

${ }^{1}$ Instituto de Fusión Nuclear, ETSI de Industriales, Universidad Politécnica de Madrid, C/ José Gutierrez Abascal, 2, E-28006 Madrid, Spain

${ }^{2}$ The Graduate School for the Creation of New Photonics Industries, 1955-1Kurematsu, Nishiku, Hamamatsu, Shizuoka, 431-1202 Japan

${ }^{3}$ Institute of Laser Engineering, Osaka University, 2-6 Yamadaoka, Suita, Osaka 565-0871, Japan

${ }^{4}$ University of Tsukuba, 1-1-1 Tennodai, Tsukuba, Ibaraki, 305-8577 Japan

${ }^{5}$ Takasaki Advanced Radiation Research Institute, Japan Atomic Energy Agency, 1233 Watanuki, Takasaki, Gunma, 370-1292 Japan

${ }^{6}$ Graduate School of Human and Environmental Studies, Kyoto University, Yoshida-Honmachi, Sakyo-ku, Kyoto, 606-8501 Japan

${ }^{7}$ Toyota Central R\&D Labs., Inc., Nagakute, Aichi, 480-1192 Japan

\section{Corresponding author:}

Raquel Gonzalez-Arrabal

Tel: +34913363110

Fax: +34913363002

e-mail: raquel.gonzalez.arrabal@upm.es 
Postal address: Instituto de Fusión Nuclear, Universidad Politécnica de Madrid

C/Jose Gutierrez Abascal 2

28006 Madid (Spain)

\begin{abstract}
The performance of a Li-ion battery (LIB) is mainly governed by the diffusion capabilities of lithium in the electrodes. Thus, for LIB improvement it is essential to characterize the lithium distribution. Most of the traditionally used techniques for lithium characterization give information about the local scale or in the macroscopic scale. However, the lithium behavior at the local scale is not mirrored at the macroscopic scale. Therefore, the lithium characterization in the mesoscopic scale would be of help to understand and to connect the mechanisms taking place in the two spatial scales. In this paper, we show a general description of the capabilities and limitations of ion beam analysis techniques to study the distributions of lithium and other elements present in the electrodes in the mesoscopic scale. The potential of the ${ }^{7} \mathrm{Li}\left(\mathrm{p}, \mathrm{\alpha}_{0}\right)^{4} \mathrm{He}$ nuclear reaction to non-invasively examine the lithium distribution as a function of depth is illustrated. The lithium spatial distribution is characterized using particle induced $\gamma$-ray $(\mu$ PIGE) spectroscopy. This technique allows estimating the density of the active particles in the electrode effectively contributing to the Li intercalation and/or de-intercalation. The advantages of the use of ion beam analysis techniques in comparison to more traditional techniques for electrode characterization are discussed.
\end{abstract}

Key words: Lithium-ion batteries, positive electrodes, Li distribution, IBA techniques, PIGE, NRA. 


\section{Introduction}

The supply and the management of energy are particularly at the centre of our dailyconcerns and stand for a socio-economic priority. Nowadays, Lithium ion batteries (LIBs) are considered to be ideal candidates for many mobile and stationary applications. In particular, the requirement to decrease the green house gas emissions produced from transport stimulates the development of Li-ion batteries to power hybrid and fully electric vehicles. They are attractive candidates for these applications as theycan provide high energy and high power densities offering the possibility to charge/discharge them many times.

The performance of a Li-ion battery is mainly governed by its energy density, power, capacity, charge and discharge rates as well as, its lifetime. Even if the Li-ion battery technology became notably better over the last two decades, technological breakthroughs seem to be necessary to further increase the energy density, the charge rate, the safety and the longevity of Li-ion batteries. Further improvements in battery development require careful clarification of the underlying physical and chemical processes, in particular, better understanding of the diffusion capabilities of the Li-ion in the electrodes. Thus, for developing advanced Li-ion batteries it is essential to use, especially in situ and non-invasive, characterization tools and methodologies, knowing the capabilities and limitations of each of them.

Modern batteries contain a wide range of metallic, organic and inorganic components with dimensions of interest ranging from local (sub-nm) up to the $\mathrm{cm}$ range. Consequently, the use of suitable techniques to investigate the different scales is crucial. However, most of the presently used techniques are oriented to obtain information about the lithium behavior in the local scale and/or in the macroscopic scale ( $\geq \mathrm{mm}$ range). As an example, the lithium atoms in a crystal have been observed by using newly developed transmission electron microscopy techniques [1] and the lithium distribution in the millimeter scale has been imaged by means of neutron radiography [2]. Though, in most cases, the lithium behavior at the local scale is not mirrored at the macroscopic scale. Therefore, the lithium characterization in the mesoscopic scale $(\mu \mathrm{m}$ 
range) would be of help to understand and to connect the mechanismstaking place in these scales.

Ion beam analysis (IBA) techniques are powerful tools which permit direct, non destructive and accurate quantitative elemental concentration characterization, including light ions such as lithium. Ion beam analysis techniques were developed in the early 1960s, as the Van de Graaff accelerators used for nuclear physics research were not able to produce the higher energies required in this field and new uses were devised for them. Thus being directly derived from nuclear physics, nowadays ion beam analysis techniques are well-established techniques widely employed in many different fields of knowledge, ranging from fundamental physics to fine arts, with special impact on materials science and solid state physics. Ion beam analysis techniques are based on the detection and analysis of the products emitted from the different processes induced when an energetic ion strikes on a target. They comprise a whole family of techniques including those based on scattering by target nuclei (Rutherford Backscattering Spectrometry, RBS, and Elastic Recoil Detection Analysis, ERDA), resonant and non-resonant nuclear reactions (RNRA and RNA, respectively), particle-induced $\gamma$-ray emission (PIGE) and particleinduced X-ray emission (PIXE), among others. A comprehensive description of ion beam analysis techniques can be found in Ref. 3.Two breakthroughs to improve the ion beam analysis techniques capabilities for material analysis arethe development of: (i) $\mu$-beam scanning procedures and (ii) of external beams. The development of $\mu$-beam scanning procedures in the 50 's has enabled extension of ion beam analysis with additional spatial information, which allows measuring the elemental distribution over specific small areas of a sample. In the $\mu$ probes, ions are raster scanned over the sample, thus enabling the extraction of $2 \mathrm{D}$ maps ofstructural and compositional information from the sample. Since the first "true" microprobe developed by Cookson and Pilling in 1970 in which they have obtained a beam spot of $15 \mu \mathrm{m}$, with $15 \mathrm{nA}$ [4], a lot of work has been carried out to improve spatial resolution by decreasing beam spot size. Nowadays, to the best of our knowledge, the spot beam size can be as small as few hundreds of nanometres, with 50-100 pA intensities [5]. 
In this paper we show a general description about the capabilities and limitations of ion beam analysis techniques to characterize the elemental composition and in particular the lithium content and distribution in the mesoscopic scale in Li-ion batteries. First, in section 2.1, we review the state of the art of the use of ion beam analysis techniques for $\mathrm{Li}$-ion batteries studies, showing some examples in which $(p, p)$ and $(p, \gamma)$ nuclear reactions have been successfully applied for lithium characterization, emphasizing their capabilities to carry lithium depthprofiling and lithium spatial distribution studies, respectively. Next, in section 2.2, we indicate the precautions needed when designing an ion beam analysis experiments for lithium characterization. In section 4.1, we illustrate the potential of the ${ }^{7} \mathrm{Li}\left(\mathrm{p}, \alpha_{0}\right)^{4} \mathrm{He}$ nuclear reaction to examine the lithium distribution, without destroying the sample. In section 4.2 , we show the capabilities of particle induced $\gamma$-ray ( $\mu$-PIGE) spectroscopy to characterize the lithium spatial distribution. In section 4.3 we discuss about the possibility of carrying out a fully 3D characterization of the lithium distribution in electrodes by using nuclear reaction analysis techniques. In sections 5 we present the advantages of using ion beam analysis techniques for the characterization Li-ion batteries over more traditional techniques. Finally in section 6 we describe the main limitations of the use of IBA for the characterization of Li-ion batteries.

\section{Nuclear reaction analysis for Li characterization in Lithium-ion Batteries}

Although the capabilities of nuclear reaction analysis techniques are amply known, to the best of our knowledge, their application to the characterization of Li-ion batteries has not been sufficiently exploited. Indeed, only few works have been devoted to this topic. For example Tadic et al. [6,7] have monitored changes in the elemental distribution at gel/polymer battery interfaces, Berger et al.[8] have characterized the elemental composition of ternary graphite-LiCa intercalation compounds, Beddy et al.[9] have characterized the lithium content of $\mathrm{LiNiVO}_{4}$ films before and after lithium insertion, Swiatowska-Mrowiecka et al. [10] have studied the dosing of intercalated lithium and determined the composition of lithiated $\mathrm{MoO}_{3}$ thin films, 
Andrade et al. [11] have measured the $\mathrm{Li}$ extraction of $\mathrm{LiMn}_{2} \mathrm{O}_{4}$ by chemical delihitation, Habrioux et al.[12] determined the lithium composition in $\mathrm{C}-\mathrm{LiFePO}_{4}$ as a function of its state of charge and. Mima et al.[13] have characterized the lithium distribution in Li-ion battery positive electrodes containing $\mathrm{Li}_{\mathrm{x}} \mathrm{Ni}_{0.8} \mathrm{Co}_{0.15} \mathrm{Al}_{0.05} \mathrm{O}_{2}$ secondary particles $(0.75 \leq \mathrm{x} \leq 1.0)$ as a function of electrode thickness and charge parameters. The main conclusion from these works is that the specific capabilities of nuclear reaction analysis techniques are very powerful to investigate $\mathrm{Li}$-ion batteries and in particular, to measure the $\mathrm{Li}$ distribution in electrodes which in combination with other techniques (X-ray absorption spectroscopy, X-ray tomography, scanning probe microscopy, transmission electron microscopy) contribute to understand the lithium intercalation/de-intercalation processes. All these works were focused on very specific aims. However, to the best of our knowledge a general description of the capabilities and limitations of nuclear reaction analysis techniques for Li-ion battery studies has not been presented so far. This is what we show in the following.

\subsection{Capabilities of the nuclear reaction techniques for $\mathrm{Li}$ characterization}

One of the key issues in Li-ion battery research is the knowledge of the lithium distribution as a function of depth, since these data allow obtaining information about the lithium diffusion in the electrodes, which is closely related to the performance of the battery (capacity, cyclability and operational charging rate).

The characterization of the lithium distribution as a function of depth is possible by using (p, p) nuclear reactions. Moreover, what is very advantageous and unique is that by using this technique such characterization can be done in a non-invasive way (see section 3.2 and Ref. [12] which may be used for in-situ characterization of the Li-ion battery.

Another important concern which needs to be addressed when studying the movement of lithium in and out of the electrode is the contribution of the active particles to the lithium intercalation/de-intercalation processes. Indeed, the differences in the lithiation/delithiation 
behavior at the local and at the macroscopic scale may be due to the fact that all the active particles in the electrode may not always effectively contribute in the same way to the lithium intercalation/de-intercalation processes. Therefore, in order to find an agreement between the macroscopic and the local scales, it is crucial to know the amount of active particles which are really contributing to the lithium motion. Thus, it is necessary to define a kind of "participation rate" which so far, to the best of our knowledge, has not been characterized.

\subsection{Peculiarities of the nuclear reactiontechniques for $\mathbf{L i}$ characterization}

In general, nuclear reaction (NRA) comprises particle-particle (p, p) reactions, particle- $\gamma(p, \gamma)$ reactions (PIGE), and particle-neutron reactions (PINE). Among all possible reactions, those available for $\mathrm{Li}$ characterization are: ${ }^{7} \mathrm{Li}\left({ }^{3} \mathrm{He}, \alpha_{0}\right){ }^{6} \mathrm{Li},{ }^{7} \mathrm{Li}\left({ }^{3} \mathrm{He}, \mathrm{d}\right){ }^{8} \mathrm{Be},{ }^{7} \mathrm{Li}\left({ }^{3} \mathrm{He}, \mathrm{p}\right){ }^{9} \mathrm{Be},{ }^{7} \mathrm{Li}\left(\mathrm{p}, \mathrm{p}_{0}\right){ }^{7} \mathrm{Li}$, and ${ }^{7} \operatorname{Li}\left(\mathrm{p}, \alpha_{0}\right){ }^{4} \mathrm{He},{ }^{7} \mathrm{Li}\left(\mathrm{p}, \mathrm{n} \gamma_{1-0}\right){ }^{7} \mathrm{Be},{ }^{7} \mathrm{Li}\left(\mathrm{p}, \mathrm{p} \gamma_{1-0}\right)^{7} \mathrm{Li}$.

The inherent peculiarities of the use of nuclear reaction techniques for materials analysis in general, and for lithium characterization in particular, force the experimentalist to take some precautions before carrying out experiments in order to achieve reliable results. These peculiarities are summarized in the following:

(i) Reaction cross-section: For a proper analysis of the results an accurate knowledge of the reaction cross-section is needed. Since no analytical theory of nuclear reaction cross sections exists, experimental data have to be used. However, most of the listed nuclear reaction cross sections were measured in the past for nuclear physics purposes and not for materials analysis. Therefore, they are often not precise enough for nuclear reaction analysis and they are sometimes available only at optimal geometrical configurations. Because of these reasons when carrying out nuclear reaction analysis experiments one has whether to carry out the experiments at those energies and detection angles for which the cross-sections exist and are well characterized, or has to characterize the reaction cross section in advance. 
Since the characterization of the reaction cross-section falls out of the scope of this work, we have identified the existing reaction cross-section for lithium characterization and we have selected the experimental conditions which allow us to make use of these existing cross sections. In particular, for PIGE experiments, the cross-section for the ${ }^{7} \mathrm{Li}\left(\mathrm{p}, \mathrm{n} \gamma_{1-0}\right)^{7} \mathrm{Be}$ and ${ }^{7} \mathrm{Li}\left(\mathrm{p}, \mathrm{p} \gamma_{1-0}\right)^{7} \mathrm{Li}$ nuclear reactions have been accurately reported by Caciolli et al. [14] for the proton beam energies in the range from 3.0 to $5.7 \mathrm{MeV}$ for a detection angle of $135^{\circ}$ to the beam direction. The cross-section for lithium characterization by using the ${ }^{7} \operatorname{Li}(\mathrm{p}, \alpha)^{4} \mathrm{He}$ nuclear reaction, which is the most suitable one for Li- depth profiling (see section 3.2), has been reported by Paneta et al.[15] for proton beam energies in the range from 1.7 to $7.0 \mathrm{MeV}$ for detection angles of $140^{\circ}$, $150^{\circ}, 160^{\circ}$ and $170^{\circ}$ to the beam direction.

(ii) Use of foils: When using ( $\mathrm{p}, \mathrm{p})$ reactions for analysis, usually the particles produced in the nuclear reaction are emitted together with backscattered particles. However, because of the difference in the cross-section, significantly smaller for the nuclear reaction production than for backscattering, the intensity of the nuclear reaction signal (yield) is much smaller than that of the backscattered particles. Therefore, in order to prevent saturation of the detector, the massive arrival of backscattered particles has to be stopped when interested in measuring the particles emerging from the nuclear reaction. For this purpose, a foil is typically placed in between the sample and the detector. Nevertheless, sometimes no foil can be used such as when the stopping power of the particles emitted in the nuclear reaction in the foil is larger than that of the backscattered particles. This is the case when using the ${ }^{7} \mathrm{Li}\left(\mathrm{p}, \mathrm{\alpha}_{0}\right)^{4} \mathrm{He}$ nuclear reaction for Li-depth profiling, since the stopping power of ${ }^{4} \mathrm{He}$ particles coming out at an energy of 7.709 $\mathrm{MeV}$ is higher than that for the protons at an energy close to $3 \mathrm{MeV}$ (incident beam). Because of the absence of a foil, special attention is needed to select the proper fluence which allows good resolution without saturating the detector. 
(iii) Occurrence of more than one nuclear reaction: Often more than one reaction is possible on a certain nucleus, resulting in the emission of different radiation and/or particles at different energies. Moreover, the presence of some other light nuclei in the material under study can also guide to nuclear reactions. These facts lead to extra peaks in the spectrum which sometimes are difficult to identify if the detailed information on the elemental composition of the samples is not known in advance. In the particular case of Li-ion batteries different reaction channels, not overlapping in energy, are opened when a proton beam impinges on a target containing lithium $\left({ }^{7} \mathrm{Li}\left(\mathrm{p}, \mathrm{p}_{0}\right){ }^{7} \mathrm{Li}\right.$, and ${ }^{7} \mathrm{Li}\left(\mathrm{p}, \mathrm{\alpha}_{0}\right)^{4} \mathrm{He}$, $\left.{ }^{7} \mathrm{Li}\left(\mathrm{p}, \mathrm{n} \gamma_{1-0}\right){ }^{7} \mathrm{Be},{ }^{7} \mathrm{Li}\left(\mathrm{p}, \mathrm{p} \gamma_{1-0}\right)^{7} \mathrm{Li}\right)$. The opening of these reaction channels leads to the appearance of diverse peaks in the spectra related to lithium, which may be useful to detect lithium, but which may overlap with any other peaks hindering the interpretation of the spectra. Moreover, apart from lithium, Li-ion batteries typically contain some other light elements like fluor and aluminum in the electrode and current collector, which lead to additional nuclear reactions (see section 3.2) whose products may eventually overlap in energy with those related to lithium. Because of these reasons detailed information on the elemental composition of the samples in advance is very useful to carry out a proper and accurate interpretation of the measured spectra.

\section{Experimental.}

\subsection{Samples.}

$\mathrm{LiFePO}_{4}$ positive electrodes were fabricated at the Graduate School of Human and Environmental Studies at the Kyoto University. These electrodes were made-up from the mixture of active material with artificial carbon and binder (polyvinyliden fluoride: PVdF). SEM images (not shown) evidence that active particles distribute randomly and homogeneously within the electrode. The average secondary active particle diameter is between 0.5 and $1.0 \mu \mathrm{m}$. 
The thickness and the areal density of the positive electrodes are $30 \mu \mathrm{m}$ and $1.0 \mathrm{mg} / \mathrm{cm}^{2}$, respectively. The porosity of the electrodes is $53 \%$.

$\mathrm{LiFePO}_{4}$ electrodes are coated on an aluminum current collector with a thickness of $25 \mu \mathrm{m}$. The electrodes were charged by attaching them to the separator. Lithium was used as the negative electrode. The current density during the charge was $45 \mathrm{~mA} / \mathrm{cm}^{2}$ and the charging time was 1 minute. Finally, the electrodes were disassembled 10 hours after charging. More details about the sample preparation procedure and characterization are reported in [16,17].

Based on the charged capacity, the average $\mathrm{Li}$ contents for the $\mathrm{LiFePO}_{4}$ electrodes after the charge is $75 \%$ of initial value.

$\mathrm{Li}_{\mathrm{x}} \mathrm{Ni}_{0.8} \mathrm{Co}_{0.15} \mathrm{Al}_{0.05} \mathrm{O}_{2}(\mathrm{LNO})$ positive electrodes were provided by Toyota Central R\&D Labs. The electrodes consist of $\mathrm{Li}_{\mathrm{x}} \mathrm{Ni}_{0.8} \mathrm{Co}_{0.15} \mathrm{Al}_{0.05} \mathrm{O}_{2}(0.75 \leq \mathrm{x} \leq 1.0)$ micrometer size particles as active material mixed with artificial carbon and binder (polyvinyliden fluoride), where the weight percentages are $85 \%$ for active material, $10 \%$ for carbon and $5 \%$ for binder. The average active particle diameter, as characterized by scanning electron microscopy (SEM) is about $5 \sim 10 \mu \mathrm{m}$. The thickness of the positive electrodes and the areal density are $105 \mu \mathrm{m}$ and $2.1 \mathrm{mg} / \mathrm{cm}^{2}$, respectively. The porosity of the electrodes was $35 \%$.

The electrodes are coated on an aluminum current collector with a thickness of $25 \mu \mathrm{m}$. For fabricating charged samples, the above positive electrode was attached to an electrolyte (mixture of dimethyl, di-ethyl, and ethyl-methyl carbonates, EC/DEC/EMC, with volume percentage of 30/40/30) and to a lithium-metal negative electrode. Then, the current was applied. The electrolyte was washed away right after charging in order to prevent Li diffusion in the electrode. More details about samples can be found in Ref. 13.

Based on the charged capacity, the average $\mathrm{Li}$ contents for the $\mathrm{Li}_{\mathrm{x}} \mathrm{Ni}_{0.8} \mathrm{Co}_{0.15} \mathrm{Al}_{0.05} \mathrm{O}_{2}$ electrodes after the charge is $75 \%$ of initial value. 
For cross-sectional studies, the electrodes were cut and their cross-sections were polished for 10 hours by using a focused argon beam at an energy of $6 \mathrm{keV}$. The beam current of the JEOL SM09010 Cross Section Polisher was $120 \mu$ A. Special precautions were taken during the cutting process to prevent the diffusion of light elements, in particular lithium. Indeed in order to be sure that the cutting process does not influence the lithium distribution, measurements were carried out for the samples cut from two opposite directions. No significant difference between them was observed.

\subsection{Li-depth profiling.}

In principle, different $(\mathrm{p}, \mathrm{p})$ nuclear reactions can be chosenfor Li-depth profiling, such as ${ }^{7} \mathrm{Li}\left({ }^{3} \mathrm{He}, \alpha_{0}\right){ }^{6} \mathrm{Li}, \quad{ }^{7} \mathrm{Li}\left({ }^{3} \mathrm{He}, \mathrm{d}\right){ }^{8} \mathrm{Be}, \quad{ }^{7} \mathrm{Li}\left({ }^{3} \mathrm{He}, \mathrm{p}\right){ }^{9} \mathrm{Be}, \quad{ }^{7} \mathrm{Li}\left(\mathrm{p}, \mathrm{p}_{0}\right){ }^{7} \mathrm{Li} \quad$ and $\quad{ }^{7} \mathrm{Li}\left(\mathrm{p}, \alpha_{0}\right){ }^{4} \mathrm{He} \quad[3,18-22]$. However, as reported by Sagara et al. [23] among them, the ${ }^{7} \mathrm{Li}\left(\mathrm{p}, \mathrm{\alpha}_{0}\right)^{4} \mathrm{He}$ is the most suitable one to carry out the analysis because of the following reasons: (i) the intensity of the signal is proportional to the amount of the isotope in the sample, thus, the naturally occurring ${ }^{7} \mathrm{Li}$ isotope is the best one for detection, (ii) it has a large Q-value (17.346 MeV) which leads to a high signal-to-noise ratio allowing the characterization of thick samples, and (iii) the reaction cross section exhibits a broad maximum at a proton energy of $\sim 3 \mathrm{MeV}[15]$ which allows estimating the depth profile directly from the energy spectrum of the $\alpha$-particles emitted in the nuclear reaction.

Then, following Sagara's work, we have characterised the Li-depth profiles by using the ${ }^{7} \mathrm{Li}(\mathrm{p}, \alpha)^{4} \mathrm{He}$ nuclear reaction. Measurements were carried out at the standard beam line of CNA at Seville University [24]. For these measurements a $\mathrm{H}^{+}$beam impinging onto the sample surface at normal incidence at an energy of $3.0 \mathrm{MeV}$ was used. The beam spot and the current were selected to be $2 \times 2 \mathrm{~mm}^{2}$ and $\sim 15 \mathrm{nA}$, respectively. Since no foil was used in between the sample and the detector, the $\alpha$-particles produced in the nuclear reaction and also the backscattered protons from other elements $(\mathrm{Fe}, \mathrm{P}, \mathrm{O}, \ldots)$ present in the electrodes were simultaneouslydetected by a Si-barrier detector with an active area of $50 \mathrm{~mm}^{2}$, located at $10 \mathrm{~cm}$ from the sample and at an angle of $150^{\circ}$. 


\subsection{Li spatial characterization.}

The elemental distribution of the samples was characterized by simultaneously measuring $\mu$ PIGE and $\mu$-PIXE spectra. The Li distribution was characterized by means of the ${ }^{7} \operatorname{Li}\left(\mathrm{p}, \mathrm{n} \gamma_{1-0}\right)^{7} \mathrm{Be}$ $\mathrm{E}_{\gamma}=429 \mathrm{keV}$ and ${ }^{7} \mathrm{Li}\left(\mathrm{p}, \mathrm{p} \gamma_{1-0}\right)^{7} \mathrm{Li} \mathrm{E}_{\gamma}=478 \mathrm{keV}$ [18] nuclear reactions. Measurements were carried out at the $\mu$-beam line of TIARA-JAEA [25] using a $\mathrm{H}^{+}$beam at an energy of 3.0 MeV. The beam current was selected to be $300 \mathrm{pA}$. The total accumulated charge per map was around 0.48 $\mu \mathrm{C}$. The beam diameter, full width at half maximum, was $1.5 \mu \mathrm{m}$. The characteristic emitted $\gamma-$ and X-rays were collected by a HPGe and by a Li-doped Si detector located at $140^{\circ}$ and at $0^{\circ}$ to the beam direction, respectively. The total scan areas were $200 \times 200 \mu \mathrm{m}^{2}$. The scanning step was 128 of the total scan range, providing $128 \times 128$ space points for each image. Measurements were carried out in high vacuum at a pressure range of $10^{-5} \mathrm{mbar}$ in order to achieve a good beam spatial resolution. Nevertheless, because of the microbeam halo effects and the lateral straggling of the beam penetrating into the samples, the lateral resolution of the microbeam scans is somewhat larger than the beam diameter. Under these conditions, typical measuring times were around 30 minutes.

$\mu$-PIGE and $\mu$-PIXE maps were estimated from the defined region of interests (ROIs) corresponding to $\mathrm{E} \gamma=478 \mathrm{keV}$ for $\mathrm{Li}$ and $\mathrm{E}_{\mathrm{x}}=7.5 \mathrm{keV}$ and $8.3 \mathrm{keV}$ for $\mathrm{Ni}-\mathrm{K}_{\alpha}$ and $\mathrm{Ni}-\mathrm{K}_{\beta}$, respectively.

\section{Results and discussion}

\subsection{Li depth profiling: Use of $(p, p)$ nuclear reactions.}

Figure 1 shows a typical ion beam spectrum for a $\mathrm{LiFePO}_{4}$ sample. The top surface of the sample, on which the proton beam was irradiated, was facing to the separator before disassembling. The two peaks observed in the high energy region of the spectra (channel 600 to 920) correspond to the $\alpha_{0}$ (left) and $\alpha_{0}$ (right) particles arising from the ${ }^{7} \mathrm{Li}\left(\mathrm{p}, \alpha_{0}\right)^{4} \mathrm{He}$ and 
${ }^{19} \mathrm{~F}\left(\mathrm{p}, \alpha_{0}\right){ }^{16} \mathrm{O}$ nuclear reactions, respectively (see inset in Fig. 1). Fluorine is not an active particle of the sample but it is contained in the binder. By coincidence the energy of the emitted $\alpha$ particles in both nuclear reactions is nearly equal: $7.709 \mathrm{MeV}$ for ${ }^{7} \mathrm{Li}$ and $7.891 \mathrm{MeV}$ for ${ }^{19} \mathrm{~F}$. Unfortunately, the overlapping of these two peaks cannot be avoided by any experimental tool and the deconvolution of the two peaks can be only done by an appropriate data analysis. The peaks appearing in the low energy region of the spectra (channels from 10 to 310) are mainly associated with backscattered protons from iron, phosphorous, oxygen and other elements present in the sample.

The distribution of the lithium concentration as a function of depth in the sample was determined by comparing the measured and calculated spectra. For the calculations the commercial computer code SIMNRA was used [26]. The cross-section input related to the lithium and fluorine nuclear reactions were taken from Paneta et al.[15] and introduced as a R33 file in the SIMNRA code.

First the spectrum was calculated by assuming that the lithium and fluorine concentration were homogeneously distributed along the whole depth. As illustrated in Fig. 2a clear discrepancies are observed between the measured and calculated spectra. In particular, the measured yield for channels between 600 and 750 which correspond to the $\alpha$-particles produced at the depths well below the sample surface is significantly higher than the calculated one. Three can be the reasons for these discrepancies: (i) the lithium concentration is not homogeneous along depth, (ii) the fluorine concentration is not homogeneous along depth, (ii) both of them are not homogeneous. However, since the distribution of the binder in the sample is homogeneous and fluorine is contained in the binder, there is no physical reason to assume that the fluorine distribution along depth is inhomogeneous. Therefore, in order to properly fit the spectrum, only the lithium distribution was assumed to be inhomogeneous along the electrode depth. To calculate the spectrum under this assumption, the sample was divided into several layers, each of them containing a different lithium concentration. Then, a run of calculations was carried out by varying the number of the layers and their lithium concentrations until a good fit was 
achieved. As depicted in Figs. $2 \mathrm{~b}$ and $2 \mathrm{c}$, the best fit was obtained by assuming that the total sample consists of nine layers, each of them containing a certain amount of lithium which increases from 4.9 atomic percentage (at. \%) for the layer close to the separator up to 6.9 at.\% for the layer close to the current collector. The layer areal density and the lithium content in each layer are schematically shown in Fig. 2c, where the left-hand side was close to the separator and the right-hand side was attached to the current collector. These results point out that the lithium concentration distribution is inhomogeneous along the electrode thickness and it rises with increasing depth from the separator.

In order to corroborate these results, the $\mathrm{LiFePO}_{4}$ electrode sample was cut (see section 3.1) and the lithium distribution was measured along the cross-section by $\mu$-PIGE. The $\mu$-PIGE Li distribution map is shown in Fig. 3a. The separator is on the upper side and the aluminum current collector in the lower side in this figure. The color relates to the lithium concentration: blue corresponds to regions with less lithium and green to regions containing more lithium. As depicted in Fig 3a and, in agreement with depth-profiling results, the lithium concentration region close to the current collector is larger (more green regions) than close to the separator. Since the iron content in each individual active particle is constant and according to SEM pictures (not shown) active particles homogeneously distribute, a clearer picture on the inhomogeneous lithium distribution along the electrode thickness can be obtained by calculating the lithium to iron ratio for every depth. To do that, the iron spatial distribution was measured by using $\mu$-PIXE with the result shown in Fig 3b. No color contrast is observed in this map indicating that, as expected, iron distributes in a homogeneous way along the whole electrode depth. Figure $3 \mathrm{c}$ shows the lithium to iron yield ratio as a function of the electrode depth, calculated after integrating the lithium and iron signals shown in Figs. 3a and 3b, respectively along the lateral direction $\left(d_{\text {lateral }}\right)$. Fig. $3 \mathrm{c}$ shows that the lithium to iron ratio gradually enhances with increasing the depth from the separator. This result is in good agreement with the results obtained when using the ${ }^{7} \mathrm{Li}\left(\mathrm{p}, \mathrm{\alpha}_{0}\right)^{4} \mathrm{He}$ nuclear reaction shown in Fig. 2c. 
Similar inhomogeneties have been observed by spectroscopy X-ray diffraction when determining the distribution of charge in $\mathrm{LiFePO}_{4}$ electrodes [27]. In this case, the inhomogeneities were attributed to a variation in the electronic resistance and to the ion conduction limit which may depend on the penetration of the electrolyte into the porous electrode. This inhomogeneity has been observed not only to depend on the electrode thickness, but also on the charge rate, the electrode elemental composition and its structure.

\subsection{Li spatial distribution characterization: Use of $(p, \gamma)$ nuclear reactions.}

The $\mu$-PIGE and $\mu$-PIXE measurements allow characterizing all elements in the samples $[3,10]$. However, for the sake of clarity, in the following we will focus only on the spatial distribution characterization of lithium and nickel. It is important to stand out that since the nickel distribution is constant (the nickel content in each individual active particle is constant and according to SEM pictures (Fig 4a) active particles homogeneously distribute within the electrode) it is going to be used as a reference signal.

Fig. 4 shows a top view scanning electron microscopy (SEM) image (a) and a $\mu$-PIGE lithium map (b), measured for a $\mathrm{Li}_{x} \mathrm{Ni}_{0.8} \mathrm{Co}_{0.15} \mathrm{Al}_{0.05} \mathrm{O}_{2}$ positive electrode. (The spatial positions in Figs. 4a and $4 \mathrm{~b}$ do not exactly correspond each other since the observed regions are different.) The diverse colors in the $\mu$-PIGE map stand for different lithium concentrations. Green color areas correspond to regions with high lithium concentration whereas blue color areas correlate with lithium depleted regions. By comparing the $\mu$-PIGE map and the SEM image we observe a similar pattern consisting of randomly distributed active particles with a size in the $\mu \mathrm{m}$ range. These results show the capabilities of $\mu$-PIGE for characterizing the lithium distribution in the mesoscopic scale.

Fig. 5a illustrates a lithium distribution map of the cross section of a $\mathrm{Li}_{x} \mathrm{Ni}_{0.8} \mathrm{Co}_{0.15} \mathrm{Al}_{0.05} \mathrm{O}_{2}$ charged positive electrode. A high color contrast is observed between the regions located close to the current collector and those located close to the separator. Since the experimental configuration was kept constant during the scan, this color contrast indicates that lithium 
inhomogeneously distributes along the electrode depth $\left(d_{d e p t h}\right)$. Principally, the lithium content is higher for regions located close to the current collector than for those far away from it. Fig. 5b shows the nickel distribution map, simultaneously measured by $\mu$-PIXE, for the same sample. Contrary to lithium, no color contrast is observed in the nickel map which indicates that, as expected, it homogeneously distributes along the whole electrode thickness. On these bases aclearer picture on the inhomogeneous lithium distribution along the electrode depth can be obtained by calculating the lithium to nickel yield ratio for every depth. Fig. 5c shows the lithium to nickel yield ratio as a function of electrode depth calculated by integrating the lithium and nickel signal intensities along the lateral direction $\left(d_{\text {lateral }}\right)$ in order to average out the particle distribution along this direction. As expected, the lithium to nickel ratio decreases with increasing distance from current collector, which definitely indicates that lithium inhomogeneously distributes along the electrode depth. This overall decrease of lithium along the depth direction is due to the movement of the lithium ions to the negative electrode through the separator during the charging process.

In addition, in Fig. 5a we observe non-uniform distribution of lithium along the lateral direction $\left(d_{\text {lateral }}\right)$. Moreover, because of the color distribution (note the region framed by a white square line in Fig. 5a), this difference is not necessarily correlated with the position of the active particle in the electrode, e. g. the distance from the current collector. This fact is better illustrated in the regions framed in white in Fig. 6 (a) and (c). These measurements were carried out in two other $\mathrm{Li}_{x} \mathrm{Ni}_{0.8} \mathrm{Co}_{0.15} \mathrm{Al}_{0.05} \mathrm{O}_{2}$ electrodes charged under the same conditions than the previously described. Here it is seen that some regions located at the same distance from the current collector but at different lateral distance $\left(d_{\text {lateral }}\right)$ exhibit a lithium content larger than that of the surrounding areas. Similar result has been previously reported by Habrioux et al.[12] who have observed that lithium distribution maps measured in charge $\mathrm{LiFePO}_{4}$ electrodes show Lirich clusters with sizes ranging from $30-60 \mu \mathrm{m}^{2}$. He tried to relate the presence of these clusters with the non-uniform charge distribution observed by Liu et al. [28] at high charging rate, concluding that since the electrodes were charged at low rate, the origin of the Li-rich clusters 
could not be due to an inhomogeneous lithium distribution during the charging process but instead it could be related to the formation of a solid electrolyte interface (SEI) layer by means of the micrometric lithium cluster growth. It is important to remark that Mima et al. have reported about inhomogeneous lithium distribution even for slow charged samples [13]. Thus, the charge rate might not be the only reason, if so, for the inhomogeneous distribution of lithium.

In our opinion, the existing of Li-rich regions in the $\mu$-PIGE maps indicates that the lithium content in diverse active particles is different, meaning that because of some reasons which need to be further investigated some of the active particles contain more lithium than others. This result may indicate that not all the active particles "participate" (are involved) in the same way in the electrochemical (lithium intercalation/de-intercalation) process. This fact may be the reason for the mismatch between data obtained in the local scale and in the macroscopic scale. Since the data obtained in the local scale may strongly depend on the observation area. Therefore, in order to have a good statistic of the system and to be able to extrapolate from the local to the macroscopic scale a lot of measurements in the local scale are needed which is a very heavy work. A different approach to find an agreement between the local and the macroscopic scales would be to measure and/or to estimate somehow the percentage of active particles effectively contributing to the lithium intercalation and to height their contribution to the electrochemical process. This is exactly the information that we can obtain from $\mu$-PIGE maps.

The knowledge of the lithium distribution in diverse active particles is very relevant, since it allows not only to nearly estimate the "participation rate" which might let to extrapolate from the local to the macroscopic scale, but also to accurately tune the size of the electrode to achieve optimal working conditions. In such a way that it allows optimizing the structure of the electrode (density of active particles, binder and conducting) and its thickness to enable the operation of the electrodes under conditions in which the maximum number of active particles are accessible to the electrochemical process. 
Finally it is remarkable to say that the inhomogeneity in the spatial lithium might depend on electrode materials and on their morphology, electrolyte type, state and type of solidelectrolyte interphase, various geometrical factors, etc. Therefore, the knowledge about the underlying processes defining lithium distribution is crucial for the manufacturing of safe, robust, and high-performance Li-ion batteries. Namely, the Li-ion battery should be designed so that the lithium distribution is kept uniform during the charge and the discharge. Thus, the present results can be applied for determining the upper limit of the electrode thickness which depends upon materials and required charge and discharge rates.

\subsection{D Li distribution characterization.}

At this point one may ask about the possibility of carrying out a fully $3 \mathrm{D}$ characterization of the lithium distribution in electrodes by using nuclear reaction analysis techniques. In principle, this could be done by combining the use of $(\mathrm{p}, \mathrm{p})$ nuclear reaction technique with $\mu$-beam scanning. However, because of the small reaction cross-section for the (p, p) nuclear reactions and the low current achieved in the $\mu$-beam setups, very long measuring times would be needed.

\section{Additional advantage of the use of IBA for Li-ion batteries characterization}

Beside the previously described ion beam analysis techniques capabilities to characterize the lithium concentration distribution, they offer some additional advantages in comparison with more traditional techniques as the ones described in the following.

\subsection{Simultaneous measurement of all elements present in the samples.}

The use of ion beam analysis technique sin an integrated approach leads to obtain information about almost all elements present in the samples. Such an approach consists of placing multiple detectors at different locations in the experimental setup enabling the simultaneous detection of all interaction products (radiation and particles) generated when an ion beam impinges into the target subjected to study. Some examples have been already mentioned in the text; while 
characterizing the lithium distributions by $\mu$-PIGE, for example, one can also obtain additional information about the concentration and distribution of other elements present in the sample by $\mu$-PIXE (Fe, Ni...).More examples are described in Ref 13 .

\subsection{Quick check of the sample homogeneity}

By using ion beam analysis techniques the homogeneity in the elemental distribution can be checked on different length scale. On one hand, as previously shown, the use of $\mu$-beams allows characterizing the elemental distribution in the $\mu \mathrm{m}$ scale. On the other hand by moving the sample in front of the beam, one can check the sample homogeneity from them up to the $\mathrm{cm}$ or even larger scales by making use of external beam facilities. As an example, Fig. 7 illustrates ion beam spectra measured for a $\mathrm{LiFePO}_{4} \mathrm{~S}$ ample at different spots separated around $2 \mathrm{~cm}$ in between them (see inset). The measuring conditions are the same as those described in section 3.2. In this figure, the peaks appearing in the low energy region of the spectra (channels from 10 to 310) are mainly associated with backscattered protons from iron phosphorus, oxygen and other elements present in the sample (RBS signal), whereas the peak located in the high energy region channel (600 to 920) correspond to the $\alpha_{0}$ particles arising from the ${ }^{7} \mathrm{Li}\left(\mathrm{p}, \alpha_{0}\right)^{4} \mathrm{He}$ and

${ }^{19} \mathrm{~F}\left(\mathrm{p}, \alpha_{0}\right){ }^{16} \mathrm{O}$ nuclear reactions (NRA signal). Clear differences are observed in the width and the height of the peaks related to the RBS signal when comparing the spectra measured at diverse spots which indicate differences in the sample thickness and elemental concentration. The differences in the peak width are further corroborated when comparing NRA spectra measured at diverse spots. These results indicate that both the elemental concentration of the electrode and its thickness are very inhomogeneous.

As previously described the use of $(\mathrm{p}, \mathrm{p})$ nuclear reactions for lithium depth profiling do not require any special sample preparation. Therefore, its use allows fast measurements and is very attractive when compared to those which are more conventional methods such (i) Augerelectron spectroscopy (AES) [29] and secondary ion mass spectrometry (SIMS) [30], in which the surface is removed by anodic oxidation or sputtering, respectively and (ii) neutron-induced 
nuclear reactions [31] which require long times for data acquisition due to limited neutron fluxes.

When comparing $(\mathrm{p}, \mathrm{p})$ nuclear reactions with similar time-scale measurements such as X-ray based methods which also offer depth resolution capabilities such as spectroscopy X-ray diffraction [27], one realizes that nuclear reactions offers important advantages. These are, among others: (i) lithium can be always detected independently if it is in crystalline or in an amorphous form, (ii) better depth resolution. In this case the main disadvantage is that ion beam analysis techniques do not offer any information about the chemical environment of lithium, information which can be deduced from X-ray based methods.

\section{Limitations of the use of IBA for Li-ion batteries characterization}

We have shown in this paper the capabilities of the use of ${ }^{7} \mathrm{Li}\left(\mathrm{p}, \alpha_{0}\right)^{4} \mathrm{He}$, of ${ }^{7} \mathrm{Li}\left(\mathrm{p}, \mathrm{n} \gamma_{1-0}\right){ }^{7} \mathrm{Be}$ and of ${ }^{7} \mathrm{Li}\left(\mathrm{p}, \mathrm{p} \gamma_{1-0}\right)^{7} \mathrm{Li}$ nuclear reactions for lithium characterization. However, it is also important to note their limitations. The main limitation is related to the maximum electrode thickness which can be analyzed. The depth limit for the analysis is mainly determined by the stopping power of ions in matter. Thus, when using the ${ }^{7} \mathrm{Li}\left(\mathrm{p}, \alpha_{0}\right){ }^{4} \mathrm{He}$ nuclear reaction for lithium depth profiling, the depth limit is dictated by the stopping power of the $\alpha$-particles in the material under study whereas, when using the ${ }^{7} \mathrm{Li}\left(\mathrm{p}, \mathrm{p} \gamma_{1-0}\right)^{7} \mathrm{Li}$ or the ${ }^{7} \mathrm{Li}\left(\mathrm{p}, \mathrm{n} \gamma_{1-0}\right)^{7} \mathrm{Be}$ nuclear reactions for the spatial characterization of lithium, the depth limit is determined by the stopping power of the impinging protons in the material under study. Since the stopping power of ions in matter depend among other factors on the material under consideration, the available depth information is not universal and has to be estimated for every particular material. The TRIM Monte Carlo [32] code allows calculating the available depth information when the elemental composition of the material under analysis and its density are known. As an example, according to TRIM calculations and assuming a typical electrode density of $2.1 \mathrm{~g} / \mathrm{cm}^{3}$, the analysis depth is limited to around $55 \mu \mathrm{m}$ when using the ${ }^{7} \mathrm{Li}\left(\mathrm{p}, \mathrm{\alpha}_{0}\right)^{4} \mathrm{He}$ and around $80 \mu \mathrm{m}$ when using ${ }^{7} \mathrm{Li}\left(\mathrm{p}, \mathrm{p} \gamma_{1-0}\right)^{7} \mathrm{Li}$. 


\section{Conclusions}

The capabilities and limitations of ion beam analysis techniques to study the elemental composition and of nuclear reactions to study the lithium distribution in the mesoscopic scale ( $\mu \mathrm{m}$ range) in Li-ion batteries have been investigated.

The use of the ${ }^{7} \mathrm{Li}\left(\mathrm{p}, \alpha_{0}\right)^{4} \mathrm{He}$ nuclear reaction allows measuring the lithium distribution as a function of depth without the necessity of any special sample preparation in a non-destructive way.

The $\mu$-PIGE technique has been demonstrated to be suitable to characterize the lithium distribution with $\mu \mathrm{m}$ spatial resolution. This technique enables rough estimation of the percentage of the active particles in the electrode effectively contributing to the lithium intercalation and/or de-intercalation. Such knowledge is very relevant since it allows designing electrodes with better properties and therefore, improving the performance of the battery.

In principle, the combination of $(\mathrm{p}, \mathrm{p})$ nuclear reactions with $\mu$-beam scanning procedures would allow fully 3D characterization of lithium distribution in electrodes. However, because of inherent peculiarities of this arrangement, very long measuring times would be needed.

The main limitation of ion beam analysis techniques for electrode characterization is related to the maximum electrode thickness which can be analyzed. The depth limit for the analysis is mainly determined by the stopping power of ions in matter.

The combination of diverse ion beam analysis techniques allow simultaneous and quick elemental characterization of all elements present in the electrode and check for the electrode homogeneity in the spatial scale which comprises from the $\mu \mathrm{m}$ up to large several hundreds of cm range.

In conclusion, ion beam analysis techniques have been demonstrated to be very well suitable to fully characterize the elemental composition of lithium-ion batteries and in particular, the lithium distribution in the mesoscopic scale which is crucial for the development of batteries with improved properties. 


\section{Acknowledgements}

The authors sincerely thank to Dr. F. J. Ferrer for his support in the NRA experiments carried out at the CNA. The authors also thank to the TIARA team for its support in the $\mu$-beam experiments. This research is supported by the Strategic International Cooperative Program

(PRI-PIBJP-2011-0810) under Ministerio de Ciencia e Innovación (MICINN) and Japan Science and Technology Agency (JST). 


\section{FIGURE CAPTIONS}

Fig. 1. Ion-beam analysis spectra for a $\mathrm{LiFePO}_{4}$ sample. The peaks appearing in the low energy region of the spectra (channels from 10 to 310) are mainly associated with backscattered protons from iron, phosphorus and oxygen and other elements present in the sample. The two peaks observed in the high energy region of the spectra (channel 600 to 920) correspond to the $\alpha_{0}$ (left) and $\alpha_{0}$ (right) particles from the ${ }^{7} \mathrm{Li}\left(\mathrm{p}, \alpha_{0}\right){ }^{4} \mathrm{He}$ and ${ }^{19} \mathrm{~F}\left(\mathrm{p}, \alpha_{0}\right){ }^{16} \mathrm{O}$ nuclear reactions (see inset).

Fig. 2. Measured (black line) and calculated (red line) NRA spectra for a $\mathrm{LiFePO}_{4}$ positive electrode. For the calculation the lithium distribution along depth was considered to be homogeneous in (a) and inhomogeneous in (b). The contributions to the calculation by lithium and fluorine are shown by the blue and green lines, respectively. Lithium content versus areal density for the best fit is shown in (c), where the left-hand side corresponds to the boundary to the separator and the right-hand side to the current collector.

Fig. 3. Lithium (a) and iron (b) distribution maps for a charged $\mathrm{LiFePO}_{4}$ positive electrode, as measured by $\mu$-PIGE and $\mu$-PIXE, respectively, and (c) lithium to iron yield ratio as a function of electrode depth calculated by integrating the area under the peaks. The upper side corresponds to the boundary to the separator and the lower side to the current collector.

Fig. 4. SEM image (a) and lithium distribution map as measured by $\mu$-PIGE (b) for a $\mathrm{Li}_{\mathrm{x}} \mathrm{Ni}_{0.8} \mathrm{Co}_{0.15} \mathrm{Al}_{0.05} \mathrm{O}_{2}$ positive electrode.

Fig. 5. Lithium (a) and nickel (b) distribution maps as measured by $\mu$-PIGE and $\mu$-PIXE, respectively for a charged $\mathrm{Li}_{x} \mathrm{Ni}_{0.8} \mathrm{Co}_{0.15} \mathrm{Al}_{0.05} \mathrm{O}_{2}$ positive electrode, and (c) lithium to nickel yield ratio as a function of electrode depth calculated by integrating the area under the peaks. The upper side corresponds to the boundary to the separator and the lower side to the current collector. 
Fig. 6. Lithium (a, c) and nickel (b, d) distribution maps as measured by $\mu$-PIGE and $\mu$-PIXE, respectively, for two charged $\mathrm{Li}_{x} \mathrm{Ni}_{0.8} \mathrm{Co}_{0.15} \mathrm{Al}_{0.05} \mathrm{O}_{2}$ positive electrodes. The white dashed lines indicate regions which exhibit high lithium content.

Fig. 7. Ion-beam analysis spectra (a) for a $\mathrm{LiFePO}_{4}$ electrode measured at different spots, randomly selected and separated around $2 \mathrm{~cm}$ in between them. (b) Photography of the sample and carton of the selected spots (color dots) in the sample where the ion beam spectra shown in (a) were measured. 


\section{REFERENCES:}

[1] R. Huang, Y.H. Ikuhara, T. Mizoguchi, S.D. Findlay, A. Kuwabara, C.A.J. Fisher, H. Moriwake, H. Oki, T. Hirayama, Y. Ikuhara, Angew. Chem.-Int. Ed. 50 (2011) 3053-3057.

[2] S. Takai, M. Kamata, S. Fujine, K. Yoneda, K. Kanda, T. Esaka, Solid State Ion. 123 (1999) 165-172.

[3] J.R. Tesmer, M.A. Nastasi, Handbook of Modern Ion Beam Materials Analysis, Materials Research Society, 1995.

[4] J.A. Cookson, Nucl. Instrum. Methods 165 (1979) 477-508.

[5] F. Watt, J.A. van Kan, I. Rajta, A.A. Bettiol, T.F. Choo, M.B.H. Breese, T. Osipowicz, Nucl. Instrum. Methods Phys. Res. Sect. B Beam Interact. Mater. At. 210 (2003) 14-20.

[6] T. Tadić, M. Jakšić, C. Capiglia, Y. Saito, P. Mustarelli, Nucl. Instrum. Methods Phys. Res. Sect. B Beam Interact. Mater. At. 161-163 (2000) 614-618.

[7] T. Tadić, M. Jakšić, Z. Medunić, E. Quartarone, P. Mustarelli, Nucl. Instrum. Methods Phys. Res. Sect. B Beam Interact. Mater. At. 181 (2001) 404-407.

[8] P. Berger, S. Pruvost, C. Hérold, P. Lagrange, Nucl. Instrum. Methods Phys. Res. Sect. B Beam Interact. Mater. At. 219-220 (2004) 1005-1009.

[9] M.V. Reddy, B. Pecquenard, P. Vinatier, C. Wannek, A. Levasseur, P. Moretto, Nucl. Instrum. Methods Phys. Res. Sect. B Beam Interact. Mater. At. 246 (2006) 397-401.

[10] J. Światowska-Mrowiecka, S. de Diesbach, V. Maurice, S. Zanna, L. Klein, E. Briand, I. Vickridge, P. Marcus, J. Phys. Chem. C 112 (2008) 11050-11058.

[11] E. Andrade, A. Romero Núñez, A. Ibarra Palos, J. Cruz, M.F. Rocha, C. Solis, O.G. de Lucio, E.P. Zavala, Nucl. Instrum. Methods Phys. Res. Sect. B Beam Interact. Mater. At. 269 (2011) 440-443.

[12] A. Habrioux, S. Surble, P. Berger, H. Khodja, A. D’Affroux, S. Mailley, T. Gutel, S. Patoux, Nucl. Instrum. Methods Phys. Res. Sect. B 290 (2012) 13-18.

[13] K. Mima, R. Gonzalez-Arrabal, H. Azuma, A. Yamazaki, C. Okuda, Y. Ukyo, H. Sawada, K. Fujita, Y. Kato, J.M. Perlado, S. Nakai, Nucl. Instrum. Methods Phys. Res. Sect. B Beam Interact. Mater. At. 290 (2012) 79-84.

[14] A. Caciolli, M. Chiari, A. Climent-Font, M.T. Fernández-Jiménez, G. GarcíaLópez, F. Lucarelli, S. Nava, A. Zucchiatti, Nucl. Instrum. Methods Phys. Res. Sect. 
B Beam Interact. Mater. At. 249 (2006) 98-100.

[15] V. Paneta, A. Kafkarkou, M. Kokkoris, A. Lagoyannis, Nucl. Instrum. Methods Phys. Res. Sect. B Beam Interact. Mater. At. 288 (2012) 53-59.

[16] Y. Orikasa, T. Maeda, Y. Koyama, H. Murayama, K. Fukuda, H. Tanida, H. Arai, E. Matsubara, Y. Uchimoto, Z. Ogumi, J. Am. Chem. Soc. 135 (2013) 54975500 .

[17] Y. Orikasa, T. Maeda, Y. Koyama, H. Murayama, K. Fukuda, H. Tanida, H. Arai, E. Matsubara, Y. Uchimoto, Z. Ogumi, Chem. Mater. 25 (2013) 1032-1039.

[18] A. Caciolli, M. Chiari, A. Climent-Font, M.T. Fernández-Jiménez, G. GarcíaLópez, F. Lucarelli, S. Nava, A. Zucchiatti, Nucl. Instrum. Methods Phys. Res. Sect. B Beam Interact. Mater. At. 249 (2006) 95-97.

[19] P. Malmberg, Phys. Rev. 101 (1956) 114-118.

[20] N. Sarma, K.S. Jayaraman, C.K. Kumar, Nucl. Phys. 44 (1963) 205-211.

[21] D. Dieumegard, B. Maurel, G. Amsel, Nucl. Instrum. Methods 168 (1980) 93103.

[22] P.D. Forsyth, R.R. Perry, Nucl. Phys. 67 (1965) 517-528.

[23] A. Sagara, K. Kamada, S. Yamaguchi, Nucl. Instrum. Methods Phys. Res. Sect. B Beam Interact. Mater. At. 34 (1988) 465-469.

[24] J. García López, F.J. Ager, M. Barbadillo Rank, F.J. Madrigal, M.A. Ontalba, M.A. Respaldiza, M.D. Ynsa, Nucl. Instrum. Methods Phys. Res. Sect. B Beam Interact. Mater. At. 161-163 (2000) 1137-1142.

[25] T. Sakai, T. Kamiya, M. Oikawa, T. Sato, A. Tanaka, K. Ishii, Int. J. PIXE 10 (2000) 91-95.

[26] M. Mayer, SIMNRA, Max Planck Institut für Plasmaphysik, n.d.

[27] H. Murayama, K. Kitada, K. Fukuda, A. Mitsui, K. Ohara, H. Arai, Y. Uchimoto, Z. Ogumi, E. Matsubara, J. Phys. Chem. C 118 (2014) 20750-20755.

[28] J. Liu, M. Kunz, K. Chen, N. Tamura, T.J. Richardson, J. Phys. Chem. Lett. 1 (2010) 2120-2123.

[29] A. Sarapulova, D. Mikhailova, L.A. Schmitt, S. Oswald, N. Bramnik, H. Ehrenberg, J. Sol-Gel Sci. Technol. 62 (2012) 98-110.

[30] J.-T. Li, J. Swiatowska, V. Maurice, A. Seyeux, L. Huang, S.-G. Sun, P. Marcus, J. Phys. Chem. C 115 (2011) 7012-7018. 
[31] G.P. Lamaze, H.H. Chen-Mayer, D.A. Becker, F. Vereda, R.B. Goldner, T. Haas, P. Zerigian, J. Power Sources 119-121 (2003) 680-685.

[32] J.F. Ziegler, M.D. Ziegler, J.P. Biersack, SRIM, n.d. 
Energy (keV)

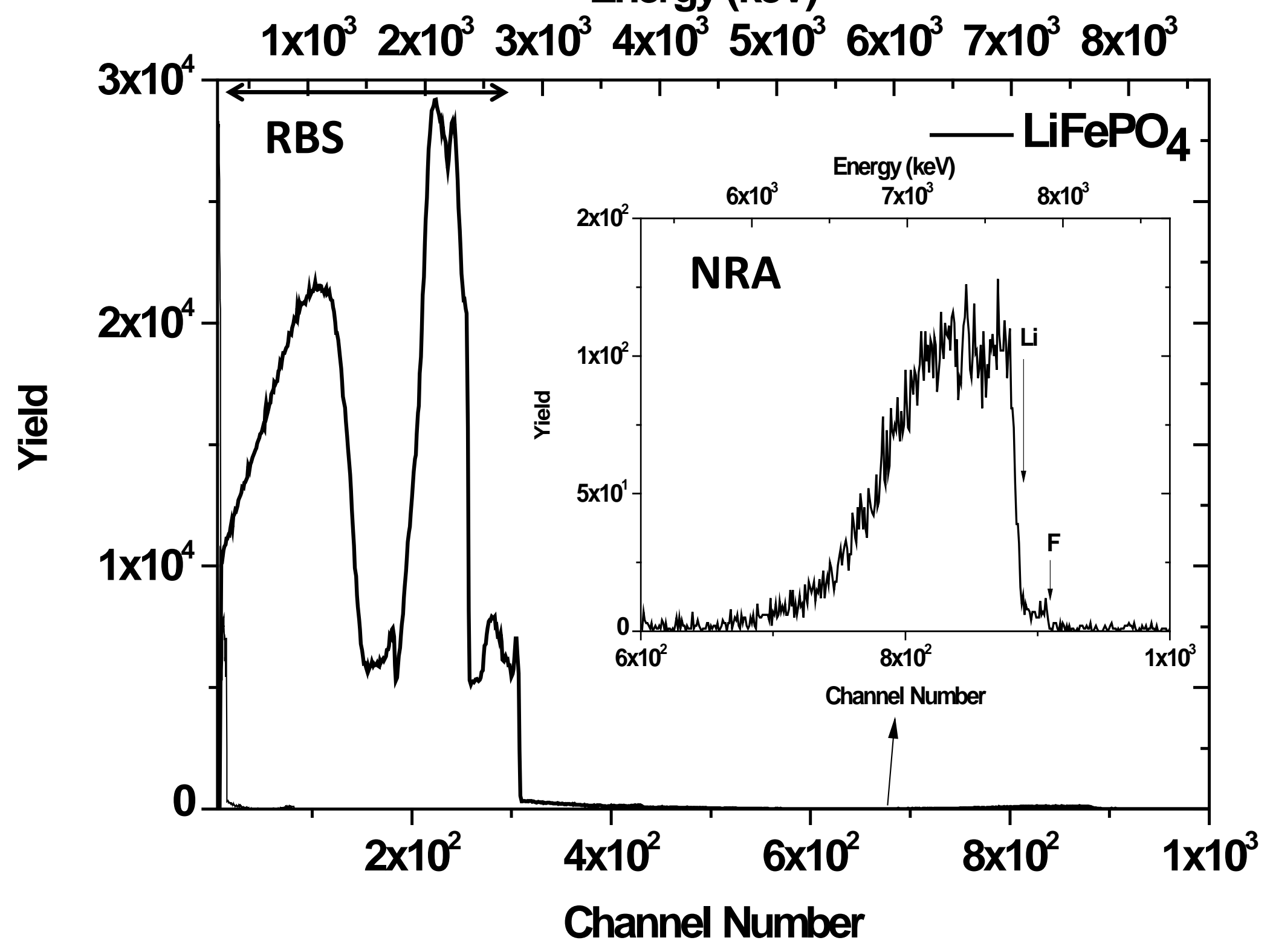

Fig. 1 Gonzalez-Arrabal et al. 

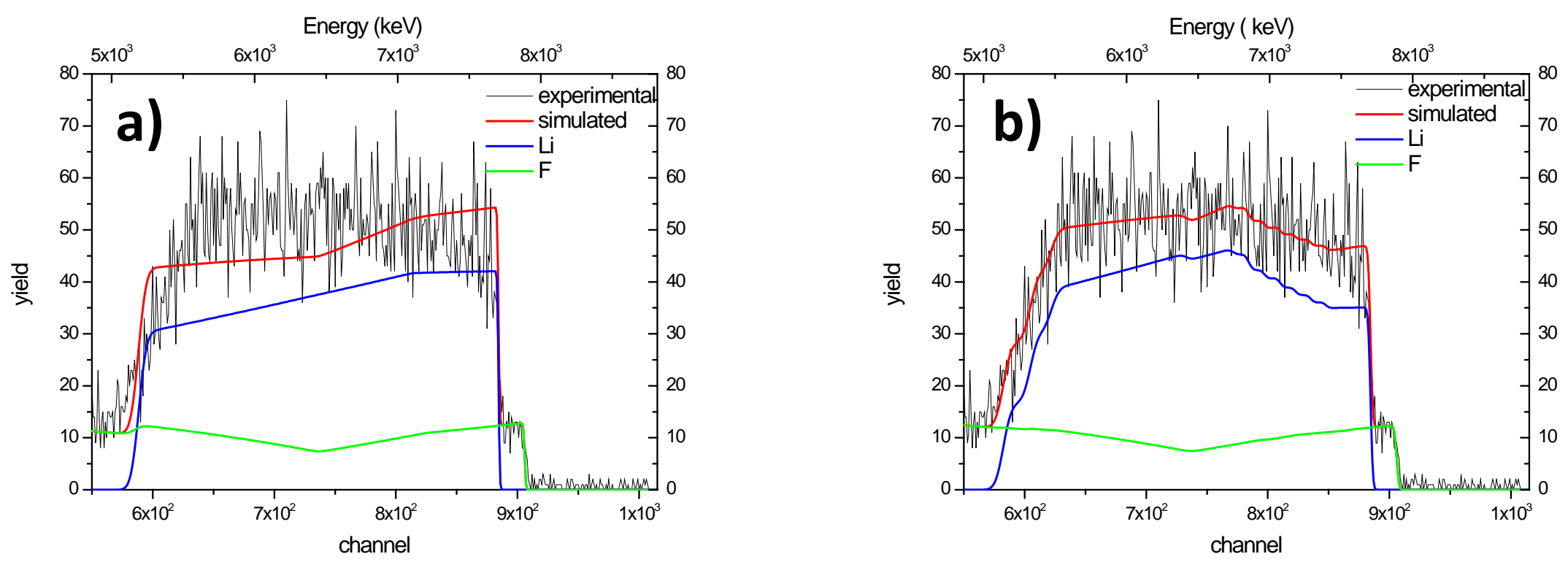

Fig. 2 Gonzalez-Arrabal et al.

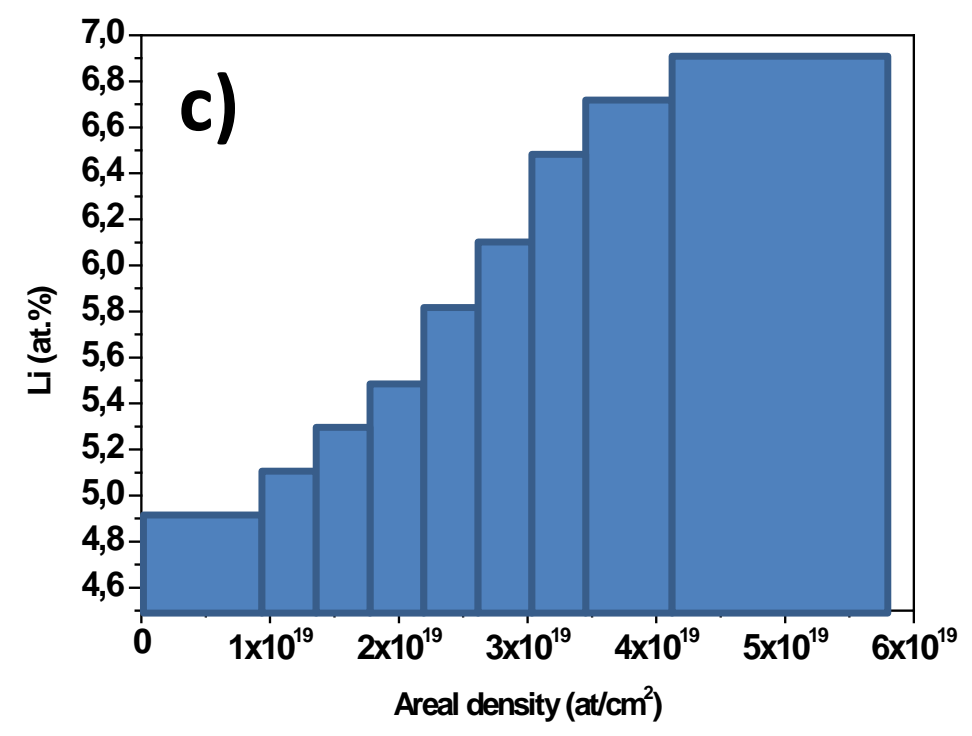




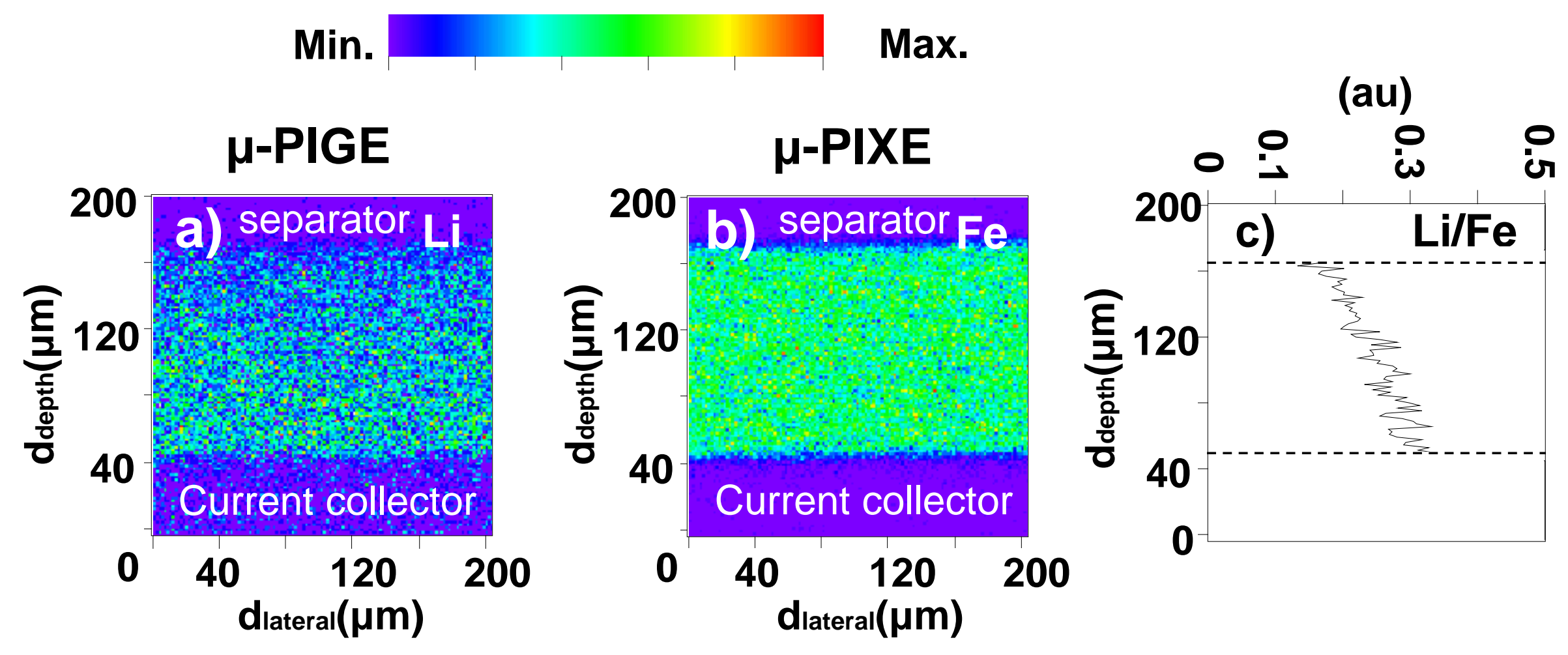

Fig. 3 Gonzalez-Arrabal et al. 


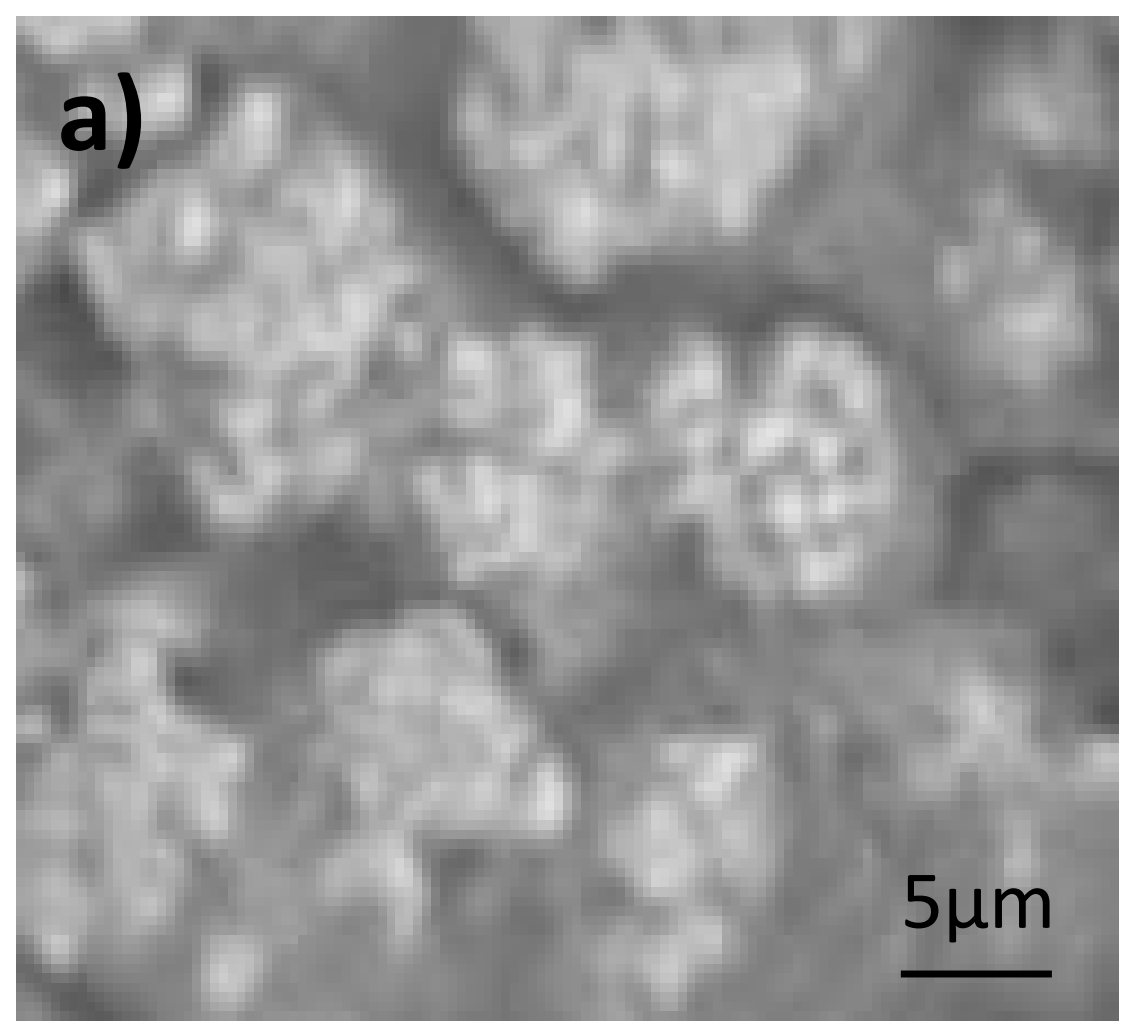

Fig. 4 Gonzalez-Arrabal et al.

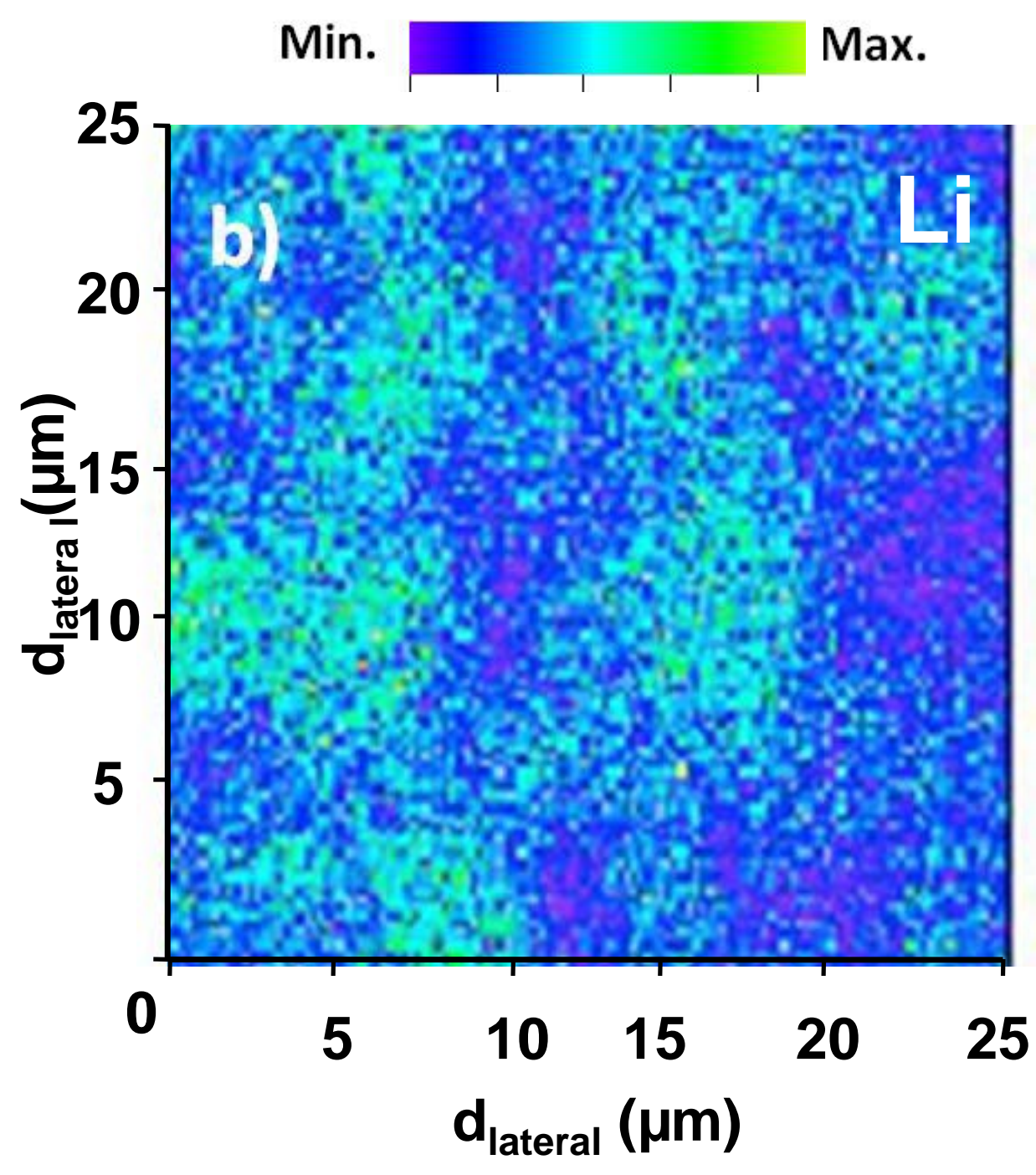




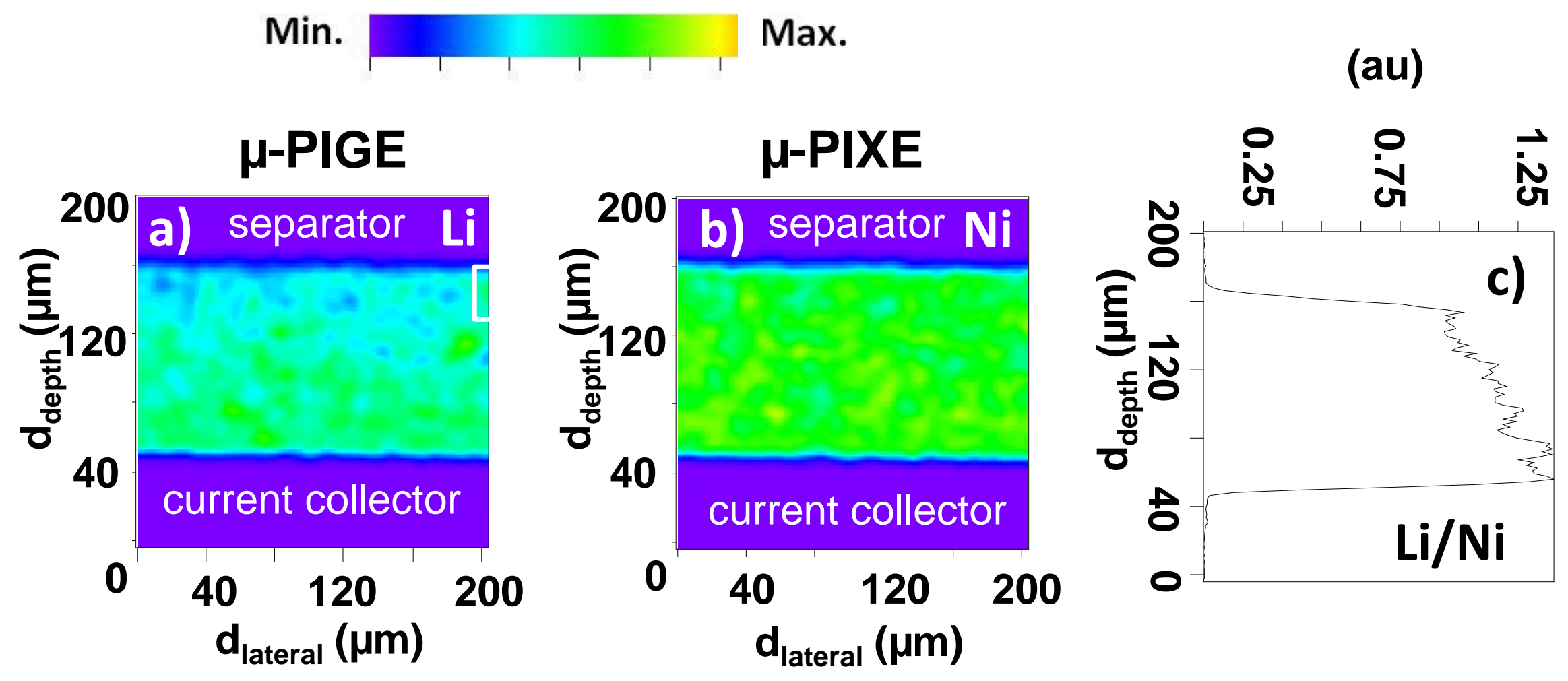

Fig. 5 Gonzalez-Arrabal et al. 
Min.

Max.
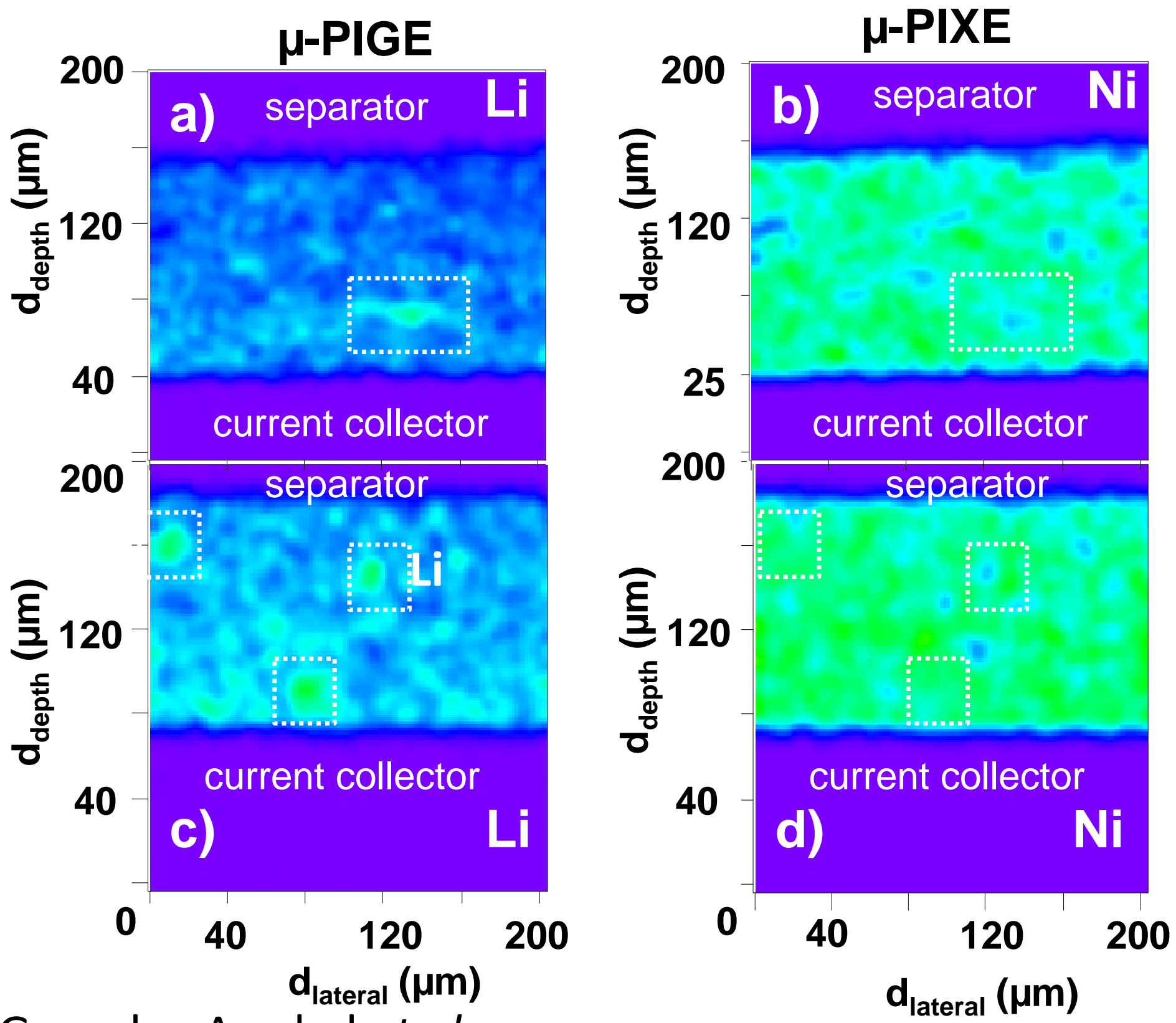

Fig. 6 Gonzalez-Arrabal et al. 


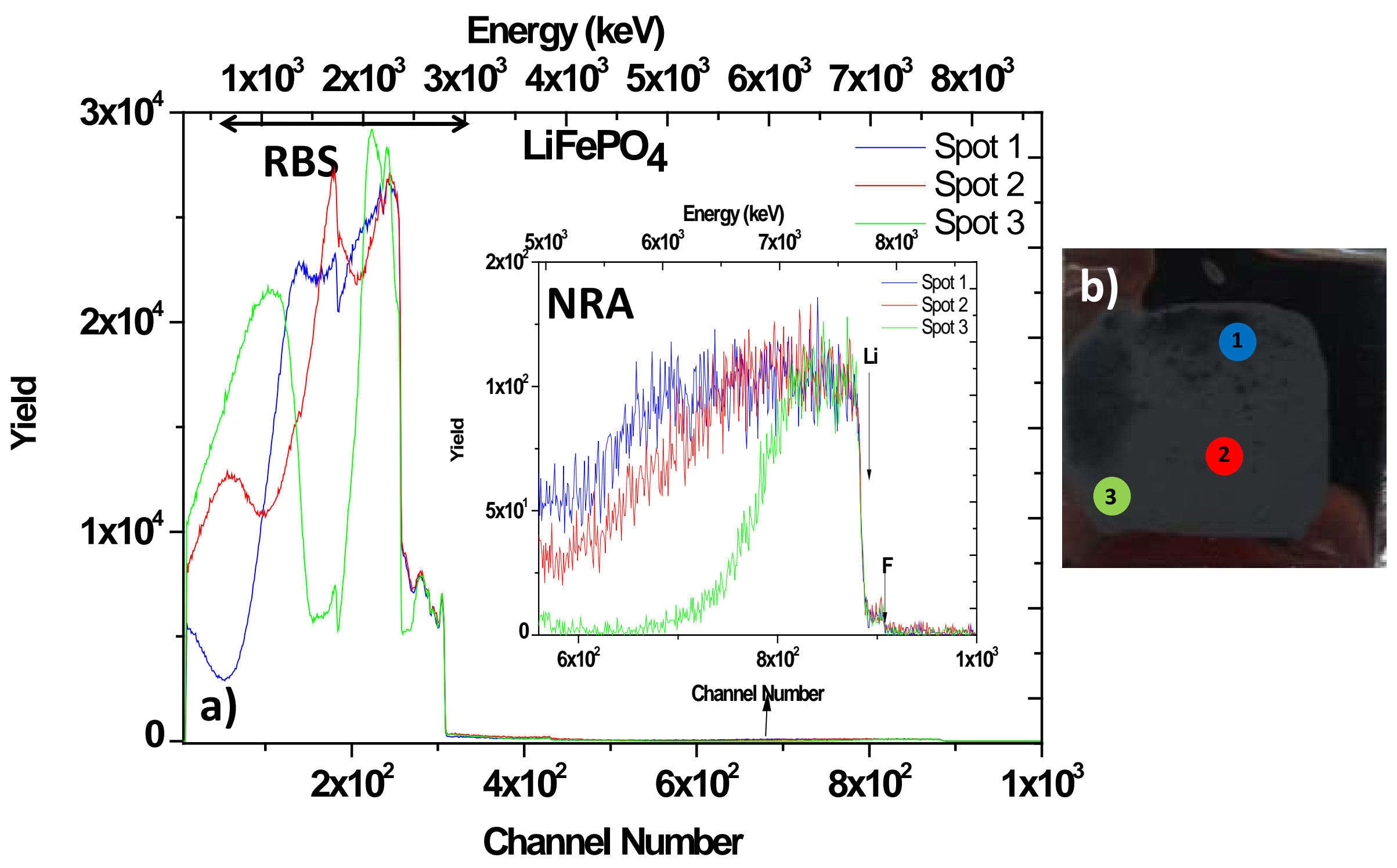

Fig. 7 Gonzalez-Arrabal et al. 